International Journal of Engineering \& Technology, $10(2)(2021) 134-138$
International Journal of Engineering \& Technology
Website: www.sciencepubco.com/index.php/IJET
Research paper

\title{
Assessing readiness for knowledge management implementation in mental hospitals, Indonesia
}

\author{
Siti Rohajawati ${ }^{1}$, Habibullah Akbar ${ }^{2}$ \\ ${ }^{1}$ Information Systems, Bakrie University, Jakarta 12920, Indonesia \\ ${ }^{2}$ Computer Science, Universitas Esa Unggul, Jakarta, Indonesia \\ *Corresponding author E-mail: siti.rohajawati@bakrie.ac.id
}

\begin{abstract}
For Southeast Asia's largest population, the prevalence of the emotional disorder has increased from 6\% in 2013 up to $9.8 \%$ in 2018 . Aligned with the e-government program, Knowledge Management (KM) offers an easier, faster, and transparent mental health services. However, the implementation relies on various factors. We conducted a workshop at 7 (seven) mental hospitals. The questionnaires were used to identify the factors that consist of awareness \& commitment, strategy, culture, structure, people, and information technology (IT). We examine the hypothesis factors of the relationship by employing the statistical analyses of correlation. This study provides at testing the relationship between factors of people, process, and technology, for KM implementation in Indonesian mental hospitals. The results of the study confirm that the relationship between Process to Technology and People has a positive effect on significance. Meanwhile, the adop-tion of the existing technical facilities have not significant support for the needs of the KM. To sum up, the study suggests further improve-ment of leadership and systems in order to serve the best of mental healthcare.
\end{abstract}

Keywords: Knowledge Management; Kendall's Coefficient; Factors Readiness; Mental Hospital.

\section{Introduction}

The life expectancy in Indonesia has improved from 63 years old in 1990 to around 70 in 2012 (until recently). This achievement is probably relating to other improvements in cases such as infant mortality rate and under-five mortality rates which are perhaps due to the economic growth that enables Indonesia to move the ladder into a middle-income country. Indeed, there are numerous health indicators that have better progress on one hand but less on the other hand. One of the most important health indicators is a mental disorder. The number of people (productive age) that experience mental disorder increases from 6\% in 2013 to $9.8 \%$ in 2018 [1]. This is due to the increasing rate of the working-age population that contributes to the difficulty for the productive age people to pursue jobs for life. At the same, economic growth also has been stagnant around 5\% in recent years. This situation is getting worse as the disparity between large cities and poorer areas have increased. The rise of mental disorder numbers seems to be related to the socio-economic factor and the pressure of rapid digital revolution that emphasizes heavily on the machine while neglecting to emotional and spiritual aspects of humans. As a result, the mental disorder may lead to internal depression, suicide, or even external outrage, violence, and abuse. At large, this may impair the society at large.

The government has developed public health facilities down to village clinics (such as 'Posyandu'), district, provincial, and up to centrallevel hospitals. However, there are remaining issues such as poor-quality infrastructures, lack of staff, and disintegration of management and financing that lead to the increasing investment by private sectors that are more difficult to maintain. The previous strategic decision to decentralize power has encouraged local flexibilities and innovation while introducing another challenge: silo culture between emerging applications, health centers, and strategic alignment between the central government and its provincial and district governments.

Recently, the Ministry of Health Indonesia launch 'Satu Data Kesehatan', an application that aimed to combine different kinds of health data such as diseases, health facilities, human resource health personnel, and budgeting. This initiation is aligned with the electronic government system which is a good indicator for developed countries. However, building an integrated system within a highly complex system is more on the social process with an incremental process [2]. Despite progress to integrate health data into the application, the mental health problem has not been receiving proper attention from the government. The mental health ecosystem and other health-related bodies such as HIV, drugs and general health has their own characteristics but may overlap and intertwined as well. It is compulsory for the mental health ecosystem to identify its own critical success factors for interoperability purposes with the national one data policy and plan.

For achieving this purpose, we believe that mental health hospitals in Indonesian need to adopt KM to improve their ability to serve and mitigate mental health-related problems that are increasing in recent years and may take a toll in the future. KM in the mental health context aims to acquire, store, manage, process, and finally share relevant and important knowledge and information in order to promote mental health in addition to preventive and curative approaches. Especially in the rapid industrial revolution that is emerging in Indonesia, major challenges in mental health may occur due to social media, digital entertainment, and possibly unemployment as a lack of projection digital 
talent in 2020-2030. Imagine, the next year 2021 more disruption technology such as augmented reality, Big Data, 3D printing, and the internet of things may hit the Indonesia job landscape. Let alone the job issue, the healthcare industry will also be disrupted as many startups as well as private and government generate Big Data that contains a huge amount of information for doctors, medical workers, and researchers in the health sector. They need to learn about people's behavior related to their mental status, new diseases, treatments, and the most effective and efficient healthcare business process. To cope with this picture, KM offers an overarching strategy to transform the traditional fragmented, inefficient healthcare system and service into more integrated and efficient that is expected to improve the quality of patient's healthcare experience. This could be achieved as such a system can present relevant knowledge to healthcare professionals and help them to make better patient care decisions.

As described previously, Indonesia as a developing country is facing multifaceted challenges in the health care ecosystem that may inhibit the effectiveness of mental health care pathways. The aim of this study is to assess the factors associated with the early readiness of mental hospital organization for KM adoption in Indonesia. The factors are consist of awareness \& commitment, strategy, culture, structure, people, and IT. Hence, we categorized into three (3) factors, i.e.; People, Process, and Technology. The study aims to find out the factors that has significant relationships to consider as a priority element for KM implementation.

\section{Knowledge management overview}

\subsection{KM as a strategic tool}

Knowledge and the KM are increasingly becoming more prominent features to achieve global competitiveness and organizational sustainability. To remain relevant, organizations need capabilities to develop, retain, organize and push employee knowledge and performances (Mårtensson, 2000; Salamah, 2011; Boh et al., 2013; Tabares et al., 2016; Haraldsdottir et al., 2018). The advancement of technology and its disruption effect requires organizations to rapidly change and prepare new business models indicating the importance of finding new ways of managing knowledge [8]. In the mental health domain, $\mathrm{KM}$ is the process of leveraging data acquired from daily activities in providing healthcare services to gain insight that helps practitioners and professionals to make good mental healthcare decisions [9]. Due to recent disrupted technologies, healthcare organizations need to have the ability to handle Big Data for mental healthcare [10]. The management of this data is extremely important as a variety of data to be processed such as unstructured social media data, video, and recorded voices. There will lots of opportunity in the tapping into the more daily basis or weekly basis into patient life in order to get deep insight why such mental disorder arise; which social or daily events that associate with a specific mental problem (schizophrenia may be related to a deep loss of loved one or severe traumatic social event; panic attack may be related to a long-term stress that accumulates over time). By visualizing such data into a dashboard with the correct selection of health indicators, type of graph, complete caption, and metainformation, the benefits of displayed knowledge will not only benefit to policymakers, professionals, and health workers but also to family, community, and society at large (Lin et al., 2015; and Hilty, 2016).

\section{2. $\mathrm{KM}$ in the mental healthcare ecosystem in Indonesia}

As a strategic tool, KM has the potential to enhance the mental health ecosystem. Refer to Orzano et al. (2008), posits the health care practice is a knowledge-based endeavor in the sense that acquisition, sharing, and organizational knowledge is not an option. Its adoption in the healthcare ecosystem may significantly enhance healthcare quality and services (Black and Sahama, 2016; and Osma et al., 2017). The ecosystem of mental healthcare in Indonesia consists of the government side, private sectors, and communities. To address the healthcare challenges, one data policy was declared by the President in June 2019. The policy includes data standardization, metadata, interoperability, and reference code. In addition to this policy, Indonesia has also committed to sustainable development goals program including job creation, overcoming disparities, poverty reduction, and services improvement. To support this complex ecosystem, KM may help to facilitate data standardization, sharing relevant data from the lowest level of healthcare facility (e.g., 'Posyandu', 'Puskesmas'= primary healthcare center), national and international bodies.

\subsection{Critical success factor for $\mathrm{KM}$ in mental healthcare}

Several success factors for adopting KM in mental health organizations have been identified by several studies. The previous research [15] found that organizational culture is on a large obstacle in KM adoption. This culture arguably is the derivative of the national culture and relates the history of a country. It can decrease the effectiveness of knowledge sharing. Thus, it is important to investigate what factors influence such culture in order to change the stable and static culture. Besides culture, the other important factors include awareness \& commitment, strategy, people, organizational structure, and IT. It was observed that reluctance to change hinder the adoption for IT system or innovation (Vest et al., 2019). The point is that there is a lack of communicated expectation for the new technology and thus the new system may not be wanted by the stakeholders. It is important in nowadays environment to utilize an agile-based development framework where stakeholders actively participate in the system development lifecycle to help with designing and evaluating the prototyped systems.

\section{Research methodology}

The main objective of this study is to examine correlations among process, people, and technology which is derived from previous research (Dubois and Tricia, 2008; Obaide and others, 2004; Rohajawati et al., 2014 \& 2016). The number of respondents is 180 persons including the head of department and staff from seven of the mental hospitals (West Java, Central Java, East Java, two from Main City of Indonesia, West Sumatera, and Nusa Tenggara Barat). The questionnaire was distributed to respondents for each mental hospital using workshop mode and then analysis used SPSS version 12. The correlation analysis was done using Kendall's and Spearman's correlation coefficient. This testing was utilized to measure the degree of relationship between two criteria (the variable $\mathrm{x}$ and $\mathrm{y}$ with which the correlation to be measured) that return value between -1 and 1 . The -1 value means there is a perfectly negative correlation while 0 means there is no correlation and 1 value means there is a perfectly positive correlation. The symbol $* *$ is used to represent a correlation that has a 0.01 significant level (2-tailed) while the symbol * is used to represent a correlation of 0.05 significant level (2-tailed). The conceptual structure is shown in Fig.1. 


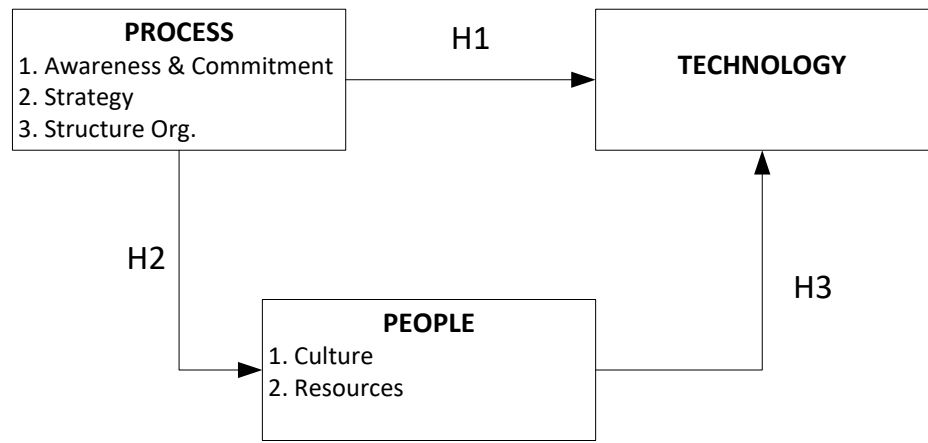

Fig. 1: The Conceptual Model in This Study.

In this study we developed the assumption for the hypothesis regarding to research question: Hypothesis $\mathrm{H}_{1}$. The relation between KM Process and KM Technology.

$\mathrm{H}_{1-1}$. The effort to establish awareness and commitment performance has a positive correlation with Technology importance.

$\mathrm{H}_{1-2}$. The effort to establish strategy performance has a positive correlation in Technology importance.

$\mathrm{H}_{1-3}$. The effort to establish structure organization performance has a positive correlation in Technology importance.

Hypothesis $\mathrm{H}_{2}$. The relation between KM Process and KM People.

$\mathrm{H}_{2-1}$. The awareness and Commitment performance has a significant correlation with People's Importance.

$\mathrm{H}_{2-2}$. The strategy performance has a significant correlation with People's Importance.

$\mathrm{H}_{2-3}$. The structure organization performance has a significant correlation with People's Importance.

Hypothesis $\mathrm{H}_{3}$. The relation between KM People and KM Technology.

H3-1. The culture performance will significantly affect Technology important.

$\mathrm{H}_{3-2}$. The resources performance will significantly affect Technology important.

The detail factors that relevant to the hypothesis are as the following:

\subsection{Awareness \& commitment}

Awareness and commitment part are intertwined and must coexist together. Awareness of what knowledge is required is the main part of the knowledge identification process. After determining the form of knowledge, the organization needs to commit and be responsible for the effectiveness of sharing identified or constructed knowledge. In our survey, these factors consist of 4 criteria: 1) KM is understanding or applying in the organization; 2) KM is representing with chief knowledge officer; 3) KM resources is demonstrating and committed by management; and 4) KM is sharing by senior.

\subsection{Strategy}

To handle the increasing trend of people with mental illness due to modern hectic life, mental health organizations need to regularly renew their strategies. To decide which strategies are relevant based on more objective assessment, they should adopt KM. In this study, this factor consists of 9 criteria: 1) KM is visioning and integrating into the business strategy; 2) KM is complying with the operational business; 3) $\mathrm{KM}$ is initiating and defining in responsibility and budget; 4) Intellectual assets are inventorying and recognizing in the organization; 5) $\mathrm{KM}$ key performance indicator is placed in line of business; 6) KM principles are establishing into procedure and guidelines; 7) KM initiative is aligning with a business plan to improve $\mathrm{KM}$; 8) $\mathrm{KM}$ is evaluating and reviewing by senior-level management; and 9) $\mathrm{KM}$ is measuring with committed as particularly of empowerment employees.

\subsection{Organizational structure}

The structure of mental health organizations provides navigation map horizontally and vertically to trace the labor division, duties sharing, and responsibilities distribution. This study surveyed 6 criteria: 1) KM resources is organizing for improving knowledge in the company; 2) KM map is structuring to point staff in direction; 3) Formal network team is existing with KM dissemination; 4) Communities of practice is establishing into an informal network in the organization; 5) Best practices idea is using positively and disseminating with rotation and mutation staff; and 6) Knowledge resource is updating and examining extensively into connection external network.

\subsection{Culture}

Rohajawati et al., (2014) suggests that individualism and the lack of cultural knowledge hinder the adoption of the KM system. Culture provides an environmentally friendly medium for everyone to share positive beliefs, norms, practices, and innovation to achieve organizational learning and continuous improvement. This survey utilizes 10 criteria: 1) Staff is learning by doing to behaviors in the company; 2) Staff is encouraging and seeing failure as an opportunity to learn; 3) Staff is getting to receive the reward for the team and share knowledge; 4) Staff is insightful to share the best way to do something; 5) Staff is helping by expert co-workers; 6) Staff is feeling secure and attractive to stay and long term employment; 7) Staff is supporting constantly to seek the best practices; 8) Staff is motivating to reflect and thinking time is allowed for; 9) Staff is facilitating by providing meeting rooms to transferring knowledge; and 10) Staff is fostering to love, care, and trust among the member of the organization.

\subsection{Resources}

Human and intellectual capitals are the key resources in mental health organizations. Even with the emergence of wide adoption of artificial intelligence in healthcare industries, people remain one of the fundamental components for sustainability. Leaders and employees must work together to encourage organizational learning and creating innovation and wisdom. In this study, this factor consists of 9 criteria: 1) KM strategies are transferring by middle level; 2) Knowledge needed is scanning and identifying by the manager; 3) Knowledge sharing 
is motivating, mentoring, and coaching by managers; 4) Staff is obeying rules in action to put their knowledge in the organization; 5) Staff is considering to able forming team with the right mix of the team by the manager; 6) Staff is feeling free to ask some help to each other; 7) Staff is training by KM program development after joining the organization; 8) KM Team is establishing to ensure the knowledge transferred internally and externally; and 9) $\mathrm{KM}$ is storing and maintaining by the specialized team.

\subsection{Technology}

The whole factors of KM relies on IT infrastructure. Mainly, the IT system is used to enable information systems that can collect, store, organize, and transfer data and information. Moreover, the IT system is also important for higher-level purposes such as knowledge exchange, foster creativity, and pushing innovation. This factor consists of 6 criteria: 1) IT is a key enabler for KM; 2) IT is available for 24/7 services; 3) Internet is available to support KM needs; 4) Standard of Procedure is available for employees; 5) Document and material are stored in the database system; and 6) IT and application are user friendly for staff.

\section{Results and discussion}

The demographic respondents are comprised of gender, age, length of work, and position. The major age group is over 38 years old as shown in Table 1. Female respondents are twice than male counterparts, more than half of the respondents have head position within their organization, and many respondents also have a length of working experiences between 11-30 years. A correlation analysis was performed to identify the factors and their factors that are most suitable for KM implementation of mental hospitals in Indonesia.

Table 1: Profile of Respondent: Gender, Age, Length of Working, and Position

\begin{tabular}{llll}
\hline Demographic characteristic & & $\mathrm{n}$ & Percentage of the total \\
\hline \multirow{2}{*}{ Gender } & Male & 82 & 45.6 \\
& Female & 98 & 54.4 \\
Age & $25-35$ & 30 & 16.6 \\
& Over 35 & 150 & 83.4 \\
\multirow{3}{*}{ Length of Working } & below 10 years & 28 & 15.5 \\
& $11-20$ years & 65 & 36.2 \\
& $21-30$ years & 69 & 38.3 \\
& 31-40 years & 18 & 10 \\
Position & Psychiatrists & 18 & 10 \\
& Doctors & 30 & 16.6 \\
& Nurses & 72 & 40.2 \\
\hline
\end{tabular}

\subsection{The relation between $\mathrm{KM}$ process and $\mathrm{KM}$ technology}

Table 2 shows the list of 3 criteria within the KM Process. According to Kendall's correlation, Awareness \& Commitment did not correspond to KM technology. A similar result was also found on Spearman. Hence hypothesis H1-1 was proven invalid. A more significant correlation of Kendall's correlation was observed between Strategy and Structure with regards to KM technology (0.085 and 0.081 respectively). However, these values were not significant enough and thus reject hypothesis H1-2 and H1-3. Similar results were also observed on their Spearman's values. This may indicate that the KM Process and its Technological infrastructure are independent in mental health hospitals.

Table 2: Kendall's and Spearman's Correlation of Between KM Process and KM Technology

\begin{tabular}{lll}
\hline \multirow{2}{*}{ KM Process } & KM Technology & Kendall $\tau$ \\
\hline Awareness \& Commitment & 0.060 & 0.074 \\
Strategy & 0.085 & 0.113 \\
Structure & 0.081 & 0.107 \\
\hline
\end{tabular}

**. Correlation is significant at the 0.01 level (2-tailed).

\subsection{The relation between $\mathrm{KM}$ process and $\mathrm{KM}$ people}

After studying the awareness and commitment, we thus study the correlation between the KM process and KM people. Table 3 shows Structure in KM Process is highly correlated to the KM based on Spearman's value (0.204) and also to (0.150) on Kendall's value. Hence, hypothesis $\mathrm{H}_{2-3}$ was proven valid. These correlations have two significant implications. The first implication is that organizational structure may induce the form of required staff and division. Second, the culture of the people that practice KM in mental health hospitals may also determine the format of the organizational structure. However, we have discovered that Awareness \& Commitment and Strategy were not correlated to KM People. The lack of correlation for both relationships were observed either for Kendall's value nor Spearman's value and thus it rejects the hypotheses $\mathrm{H}_{2-1}$ and $\mathrm{H}_{2-2}$.

Table 3: Kendall's and Spearman's Correlation of Between KM Process and KM People

\begin{tabular}{|c|c|c|}
\hline KM Process & $\begin{array}{l}\text { KM People } \\
\text { Kendall } \tau \\
\end{array}$ & Spearman $\rho$ \\
\hline Awareness \& Commitment & 0.037 & 0.044 \\
\hline Strategy & 0.080 & 0.112 \\
\hline Structure & $0.150 * *$ & $0.204 * *$ \\
\hline
\end{tabular}

**. Correlation is significant at the 0.01 level (2-tailed).

\subsection{The relation between KM people and KM technology}


The correlation assessment between KM People and KM Technology suggests that Culture within KM People were not correlated to KM Technology as shown on Table 4. These results were obvious as Kendall's value is 0.048 and the Spearman's value is 0.069 . More surprisingly, similar results also found between Human Resources and KM Technology ( 0.096 and 0.132 for Kendall and Spearman respectively). This shows a bad indication that the developed facilities have not been integrated to the need of the KM People. Hence hypotheses $\mathrm{H}_{3-1}$ and $\mathrm{H}_{3-2}$ were proven invalid.

Table 4: Kendall's and Spearman's Correlation of Between KM People and KM Technology

\begin{tabular}{lll} 
& Table 4: Kendall's and Spearman's Correlation of Between KM People and KM Technology \\
\hline \multirow{2}{*}{ KM People } & KM People & Kendall $\tau$ \\
& 0.048 & 0.069 \\
\hline Culture & 0.096 & 0.132 \\
Human Resources & & \\
\hline ** Correlation is significant at the 0.01 level (2-tailed) &
\end{tabular}

Our results interestingly showed that there is a fundamental problem of the developed technological facilities within mental health hospitals/organizations that we have surveyed. The results indicated that these facilities have not been developed based on the demand of the KM Process and KM People. In order to support the increasing trend of the number of patients with a mental disorders, organizations must align and modify their technological facilities to enable more mature KM adoption.

\section{Conclusion}

This study suggests the priority factor for implementing KM in mental hospitals in order to provide the superfine of healthcare services. Firstly, KM Process and KM Technology. Secondly, KM Process and KM People, and third between KM People and KM Technology. In the first and second relationships, it is clearly showed that the adoption of the existing technological facilities has not supported the needs of the KM People and Process. However, the results also indicated that there is already a correlation between KM People and Process i.e. for the organizational structure and the people. To provide a better adoption of KM, we mainly suggest two pieces of advice based on the findings. Firstly, the policy and decision should be more oriented to the demand of middle-level and operational staff. Secondly, mental health hospitals need to focus more on knowledge sharing horizontally and thus maximizing the use of the IT systems.

\section{Acknowledgement}

This work has been funded by Bakrie University and Directorate of Higher Education - Ministry of Research and Technology through grant Applied Research of Higher Education (PTUPT), Indonesia. We would also like to thank the Indonesian Psychiatrists Association. Special thanks to our colleagues; dr. Eka Viora, SpKJ; dr. Diah Setia Utami, SpKJ, MARS (PDSKJI); dr. Bambang Eko Sunaryanto, SpKJ, MARS (ARSAWAKOI); and all respondents of the research. Thank you also to directors of mental hospitals in Indonesia (Jakarta, Cibubur, Bogor, Magelang, Lawang, Padang, dan Mataram).

\section{References}

[1] Kemenkes RI, "Hasil Utama Riskesdas 2018," https://www.persi.or.id/images/2017/litbang/riskesdas_launching.pdf, 2018.

[2] J. Braa, S. Sahay, J. Lewis, and W. Senyoni, "Health information systems in indonesia: understanding and addressing complexity," in IFIP International Federation for Information Processing, ICT4D 2017, 2017, pp. 59-70. https://doi.org/10.1007/978-3-319-59111-7 6.

[3] M. Mårtensson, “A critical review of knowledge management as a management tool,” J. Knowl. Manag., vol. 4, no. 3, pp. 204-216, 2000, https://doi.org/10.1108/13673270010350002.

[4] U. Salamah, “Conceptual framework knowledge management implementation relationship in shaping organizational competitive advantage,” J. Appl. Sci. Res., vol. 7, no. 8, pp. 1315-1319, 2011

[5] W. F. Boh, T. T. Nguyen, and Y. Xu, "Knowledge transfer across dissimilar cultures," J. Knowl. Manag., vol. 17, no. 1, pp. 29-46, 2013, https://doi.org/10.1108/13673271311300723.

[6] M. S. Tabares, L. Giraldo, and L. Joyanes, "Improving the business processes management from the knowledge management," ACM Int. Conf. Proceeding Ser., vol. Part F1305, 2016, https://doi.org/10.1145/2925995.2925998.

[7] R. K. Haraldsdottir, J. Gunnlaugsdottir, E. T. Hvannberg, and P. Holdt Christensen, "Registration, access and use of personal knowledge in organizations," Int. J. Inf. Manage., vol. 40, no. January, pp. 8-16, 2018, https://doi.org/10.1016/j.ijinfomgt.2018.01.004

[8] K. North and G. Kumta, "Knowledge management: Value creation through organizational learning," BOOK, 2018.

[9] J. Osma, M. Sprenger, and T. Mettler, "Introduction of e-mental health in national health systems - A health professionals' perspective," Heal. Policy Technol., vol. 6, no. 4, pp. 436-445, 2017, https://doi.org/10.1016/j.hlpt.2017.07.001.

[10] N. Elgendy and A. Elragal, "Big Data Analytics in Support of the Decision Making Process,” Procedia Comput. Sci., vol. 100, pp. 1071-1084, 2016, https://doi.org/10.1016/j.procs.2016.09.251.

[11] W. Lin, W. Dou, Z. Zhou, and C. Liu, “A cloud-based framework for Home-diagnosis service over big medical data,” J. Syst. Softw., vol. 102, pp. 192-206, 2015, https://doi.org/10.1016/j.jss.2014.05.068.

[12] D. M. Hilty and D. Mucic, "Technology, health, and contemporary practice: How does e-mental health fit it and what does it offer?," e-Mental Heal., pp. 3-27, 2016, https://doi.org/10.1007/978-3-319-20852-7_1.

[13] A. John Orzano, C. R. McInerney, D. Scharf, A. F. Tallia, and B. F. Crabtree, "A knowledge management model: Implications for enhancing quality in health care," J. Am. Soc. Inf. Sci. Technol., vol. 59, no. 3, pp. 489-505, 2008, https://doi.org/10.1002/asi.20763.

[14] A. S. Black and T. Sahama, "Chronicling the patient journey: Co-creating value with digital health ecosystems," ACM Int. Conf. Proceeding Ser., vol. 01-05-Febr, 2016, https://doi.org/10.1145/2843043.2843381.

[15] S. Rohajawati, D. I. Sensuse, Y. G. Sucahyo, and A. M. Arymurthy, "Mental health knowledge management: critical success factors and strategy of implementation," J. Knowl. Manag., vol. 20, no. 5, pp. 980-1003, 2016, https://doi.org/10.1108/JKM-10-2015-0378.

[16] S. Rohajawati, A. Sugiana, D. I. Sensuse, Y. G. Sucahyo, and S. Lusa, "Identifying components knowledge management for e-Health (Case study: Mental Hospital, Indonesia)," in 2014 2nd International Conference on Technology, Informatics, Management, Engineering \& Environment, 2014, pp. 200-205. https://doi.org/10.1109/TIME-E.2014.7011618. 